\title{
Comparison of three ultrasound based elastographic techniques in children and adolescents with chronic diffuse liver diseases.
}

\author{
Oana Belei ${ }^{1}$, Ioan Sporea², Oana Gradinaru-Tascau², Laura Olariu ${ }^{1}$, Alina Popescu², \\ Ioan Simedrea $^{1}$, Otilia Marginean ${ }^{1}$
}

${ }^{1}$ Ist $^{\text {Pediatric Department, }}{ }^{2}$ Gastroenterology and Hepatology Department, University of Medicine and Pharmacy Victor Babes, Timisoara, Romania

\begin{abstract}
Non-invasive techniques for liver fibrosis assessment were developed for adults and recent researches tested their accuracy in children. The only validated elastographic method for non-invasive liver fibrosis evaluation in children is Transient Elastography (TE). The aim of our study was to investigate the feasibility of liver stiffness (LS) measurement in paediatric patients with chronic liver diseases by means of Acoustic Radiation Force Impulse Elastography (ARFI) and 2D-Shear Wave Elastography (2D-SWE), compared to TE as reference method. Material and methods: We enrolled 54 consecutive children and adolescents with different chronic liver diseases. All patients were examined by means of TE, ARFI, and 2D-SWE. All measurements were performed in the right liver lobe, in the same session, in fasting condition. We considered reliable LS elastographic measurements as follows: for TE and ARFI - the median value of ten measurements with a success rate $\geq 60 \%$ and an interquartile range $<30 \%$, for 2D-SWE - the median value of five measurements. Results: The successful measurement rate for TE was 94.4\% (51/54). Taking TE as a reference method, sensitivity of ARFI for detecting fibrosis F1 was $71.42 \%$, for F2-77.77\%, for F3-62.5\% and for F4-71.42\%. Sensitivity of 2D-SWE for detecting F1 was 92.85\%, for F2-83.33\%, for F3-87.5\% and for F4-85.71\%. We found significant correlations between TE and 2D-SWE on the entire lot (Kappa correlation factor $=0.843, \mathrm{p}=0.001)$. Analyzing the subgroup with $\mathrm{SR}=60 \%-70 \%$, we did not find significant correlation between TE and ARFI (Kappa correlation factor $=0.172, \mathrm{p}=0.452$ ). Assessing the subgroup with $\mathrm{SR}>70 \%$, we found a significant correlation between TE and ARFI (Kappa correlation factor $=0.761, \mathrm{p}=0.001$ ). Conclusions: Overall, 2D-SWE correlate better with TE compared to ARFI in children. Excluding patients with less satisfactory technical parameters, we obtained significant correlations between all three methods. Both SWE and ARFI are non-invasive techniques feasible of performing on paediatric patients along with TE.

Keywords: children, liver stiffness, Transient Elastography, Acoustic Radiation Force Impulse Elastography, 2D-Shear Wave Elastography.
\end{abstract}

\section{Introduction}

Fibrosis is a progressive process that appears as a consequence of liver injuries in the majority of chronic liver diseases, including chronic $\mathrm{B}$ and $\mathrm{C}$ viral hepatitis, metabolic liver diseases such as Wilson disease or hemochromatosis, billiary atresia, or nonalcoholic stea-

Received 19.10.2015 Accepted 10.01.2016

Med Ultrason

2016, Vol. 18, No 2, 145-150

Corresponding author: Oana Belei

4/17 Heinrich Heine Street

300041 Timisoara, Romania

Phone: 0040723289480

E-mail: oana22 99@yahoo.com to-hepatitis. Early detecting of liver fibrosis can significantly improve the prognosis of paediatric patients [1]. It is recognised that liver biopsy is the gold standard for diagnosis of fibrosis, but biopsy is an invasive technique and may have a potential risk of complications such as bleeding [2]. Also, the tissue biopsy specimen can be too small to obtain reliable results [3]. Besides sampling error, there can be interobserver variation in staging [4].

Ultrasonography based elastographic methods that are expected to replace liver biopsy have recently been developed, including transient elastography (TE), acoustic radiation force impulse (ARFI), real-time tissue elastography (RTE), and two-dimensional real-time shear wave elastography (2D-SWE) [5]. 2D-SWE is the new- 
est of these methods and has the following advantages compard to TE: real-time 2D imaging, simple operation, qualitative (color coded) and quantitative measurements, expressed in meter/second (m/s) or kiloPascals (KPa) [6].

Non-invasive techniques for liver stiffness (LS) assessment were developed for adult patients, but recent research has tested their accuracy in children. Liver biopsy is not very well accepted by the parents of children with chronic hepatitis and it is difficult to use it during the follow-up in case of paediatric patients. There is a trend towards replacing liver biopsy by elastography for the evaluation of liver fibrosis among children with chronic diffuse liver diseases [7,8].

Recent meta-analyses $[9,10]$ have reported that TE is a reliable diagnostic tool for the non-invasive evaluation of liver fibrosis in patients with chronic liver conditions and is a validated method for LS evaluation both in children and adults [11]. The advantage of TE is its non-invasiveness, which makes it possible to perform repeated liver fibrosis assessments on a large number of asymptomatic patients in a cross-sectional and serial fashion [12]. The principle of ARFI is that shearing of the examined tissue induces a strain into the tissues [13,14]. Few recent paediatric studies have set the standard ARFI elastography values for liver in healthy children $[15,16]$ only defining normal liver ARFI values in children, without discriminating between the different degrees of liver fibrosis in paediatric patients. 2D-SWE is based on Supersonic Imaging (SSI), an ultrasound-based technique used for real-time visualization of soft tissue viscoelastic properties [6]. Due to paucity of researches on this topic in the paediatric population, no validated specific cut-off range values for ARFI and 2D-SWE have been established in order to discriminate fibrosis degree among children with different liver diseases.

The aim of this paper was to investigate the feasibility of LS measurement in children and adolescents with chronic diffuse liver diseases by means of ARFI and 2DSWE, compared to TE as reference method. This study assessed the correlations between three elastographic methods: TE, ARFI and 2D-SWE, with respect to their practical use in paediatric patients, attempting to validate alternative elastographic techniques with similar accuracy to TE in children.

\section{Material and methods}

\section{Study design}

The study was conducted at the "Louis Turcanu" Emergency Hospital for Children in Timisoara and theGastroenterology Unit of Emergency County Hospital Timisoara, Romania. The study group consisted of 54 consecutive children and adolescents admitted with different chronic liver diseases: 14 with chronic B hepatitis, 10 chronic $\mathrm{C}$ hepatitis, 5 autoimmune hepatitis, 19 nonalcoholic steato-hepatitis, 4 Wilson disease, 2 juvenile hemochromatosis. LS was measured for each subject using three elastographic methods in the same session: TE, ARFI and 2D-SWE.

All legal guardians/parents of patients enrolled in this study signed an informed consent form prior to inclusion. Institutional ethical approval was obtained for this work.

Inclusion criteria were: paediatric patients with chronic liver diseases aged between 6 and 18 years old, (mean age $8.6 \pm 2.5$ years), weighing over $20 \mathrm{~kg}$ and with thorax perimeter $>45 \mathrm{~cm}$. These somatic parameters were set as inclusion criteria in order to obtain reliable results by FibroScan according to the manufacture's indication for using M probe. All subjects had normal or slightly elevated aminotransferases levels. Exclusion criteria were: patients with elevated aminotransferases level $(>3$ times upper limit of normal values), significant cholestasis, decompensated cirrhosis and focal liver lesions.

For all patients LS was measured by means of TE using a FibroScan device (EchoSens, Paris, France). For each patient 10 valid TE measurements were performed using $\mathrm{M}$ or XL probe according to the body mass index (BMI) and the median value was calculated. Reliable measurements were defined as: median value of 10 valid LS measurements with a success rate (SR) $\geq 60 \%$ and an interquartile range interval $(\mathrm{IQR}) \leq 30 \%$. SR represents the ratio of the number of successful acquisitions over the total number of acquisitions and IQR represents the difference between the 75th and 25th percentile, essentially the range of the middle $50 \%$ of the data.

ARFI was performed with a Siemens Acuson S2000 Virtual Touch ultrasound system (Siemens AG, Erlangen, Germany) with a 4CI probe. The measurements were performed at two $\mathrm{cm}$ under the liver capsule. Ten valid ARFI measurements were performed and the median value was calculated. Even if the manufacturer did not recommend for ARFI the use of technical parameters IQR and SR well-known in TE, based on a published paper from our group [2], we considered for ARFI those measurements with a $\mathrm{SR} \geq 60 \%$ as reliable determinations in this study .

2D-SWE was performed with an Aixplorer ${ }^{\mathrm{TM}}$ ultrasound (SuperSonic Imagine, Aix-en-Provence, France), using a SC6-1 convex probe. The median value of 5 valid measurements was calculated. The measurement was performed 1-2 cm under the liver capsule. Results were expressed in $\mathrm{KPa}$.

All measurements were performed under fasting conditions, with minimal scanning pressure applied by the operator in the right liver lobe, in supine position 
Table I. The cut-off range values for fibrosis staging used in this study for each elastographic technique $[11,15,17]$.

\begin{tabular}{llll}
\hline Fibrosis staging & TE (KPa) & ARFI (m/s) & 2D-SWE (KPa) \\
\hline F0 & $2.5-5.9$ & $0-1.19$ & $0-7.39$ \\
F1 & $6-7.19$ & $1.2-1.39$ & \\
F2 & $7.2-9.59$ & $1.4-1.59$ & $7.4-8.69$ \\
F3 & $9.6-14.49$ & $1.6-1.99$ & $8.7-9.19$ \\
F4 & $>14.5$ & $>2$ & $>9.2$ \\
\hline
\end{tabular}

TE - Transient elastography, ARFI - Acoustic Radiation Force Impulse Elastography, 2D-SWE - 2D-Shear Wave Elastography

by intercostal approach, with the right arm in maximum abduction, in the same session. The measurements were performed by two highly skilled operators with experience in adult patients.

The cut-off range values for fibrosis staging used in this study for each elastographic technique according to the most recently published studies are shown in table I $[11,15,17]$.

\section{Statistical analysis}

The statistical analysis was performed using SPSS17 software. Means and standard deviations (SD) were calculated for numerical variables with a normal distribution, whereas for variables with a non-normal distribution, medians and ranges were calculated. Qualitative variables were expressed as numbers and percentages. Kappa Statistic tests were computed in order to assess correlations between variables. Chi ${ }^{2}$ test (with Yates' correction for continuity) was used to compare proportions expressed as percentages. Ninety-five percent confidence intervals $(95 \% \mathrm{CI})$ were calculated for each predictive test. A p-value $<0.05$ was considered as indicating significance for each statistic test.

\section{Results}

From 54 children assessed by TE using FibroScan, in 51 cases we obtained valid measurements with $\mathrm{SR} \geq 60 \%$ and $\mathrm{IQR} \leq 30 \%$. The proportion of valid measurements by means of TE using FibroScan was 94.4\% (51/54 patients). Three cases were obese children with BMI $>25 \mathrm{~kg} / \mathrm{m}^{2}$ and although we used XL probe, the results were indeterminable. These cases were excluded from further analyses.

All 51 patients with valid TE measurements had also valid results when performing ARFI and 2D-SWE. For all ARFI measurements we obtained $\mathrm{SR}=100 \%$ and IQR $<30 \%$. The feasibility for $2 \mathrm{D}-\mathrm{SWE}$ was $100 \%$.

According to TE measurements, from 51 paediatric patients examined, 4 patients had no fibrosis (F0), 14 cases had F1, 18 cases had F2, 8 cases had F3 and 7 cases had cirrhosis - F4. The mean values for each elastographic technique are shown in table II.
Table II. The mean values of the elastographic technique obtained in the study group.

\begin{tabular}{ll}
\hline Technique & Mean values \\
TE & $9.29 \pm 3.95 \mathrm{kPa}, 95 \% \mathrm{CI}[8.18-10.40]$ \\
ARFI & $1.50 \pm 0.40 \mathrm{~m} / \mathrm{s}, 95 \% \mathrm{CI}[1.39-1.62]$ \\
2D-SWE & $7.76 \pm 2.46 \mathrm{kPa}, 95 \%$ CI [7.07 - 8.46] \\
\hline TE - Transient elastography, ARFI - Acoustic Radiation Force Im- \\
pulse Elastography, 2D-SWE - 2D-Shear Wave Elastography
\end{tabular}

Table III. The specificity and sensitivity for ARFI and 2D-SWE in staging hepatic fibrosis.

\begin{tabular}{|c|c|c|c|c|}
\hline \multirow{2}{*}{$\begin{array}{l}\text { Method } \\
\text { Fibrosis stage }\end{array}$} & \multirow{2}{*}{$\begin{array}{l}\text { ARFI } \\
\text { Sp }\end{array}$} & \multicolumn{3}{|c|}{ 2D-SWE } \\
\hline & & Sn & Sp & Sn \\
\hline F1 & $89 \%$ & $71.42 \%$ & $89.18 \%$ & $92.85 \%$ \\
\hline F2 & $96.96 \%$ & $77.77 \%$ & $96.96 \%$ & $83.33 \%$ \\
\hline F3 & $100 \%$ & $62.5 \%$ & $100 \%$ & $87.5 \%$ \\
\hline $\mathrm{F} 4$ & $100 \%$ & $71.42 \%$ & $100 \%$ & $85.71 \%$ \\
\hline
\end{tabular}

$\mathrm{Sp}$ - specificity, Sn - sensitivity, ARFI - Acoustic Radiation Force Impulse Elastography, 2D-SWE - 2D-Shear Wave Elastography

Table IV. Kappa correlation factor between TE/ARFI and TE/2D-SWE

\begin{tabular}{ll}
\hline Kappa factor TE/ARFI & $0.683, \mathrm{p}=0.001$, \\
& $99 \% \mathrm{CI}[0.000-0.001]$ \\
Kappa factor TE/2D-SWE & $0.843, \mathrm{p}=0.001$, \\
& $99 \% \mathrm{CI}[0.000-0.001]$ \\
\hline
\end{tabular}

TE -Transient elastography, ARFI - Acoustic Radiation Force Impulse Elastography, 2D-SWE - 2D-Shear Wave Elastography

The specificity $(\mathrm{Sp})$ and sensitivity $(\mathrm{Sn})$ for detecting each stage of fibrosis for both methods ARFI and 2DSWE are detailed in table III.

The Sn for detecting F1 and F2 for 2D-SWE elastography were significantly higher than ARFI technique $(p<0.05)$. Also, Sn of 2D-SWE for detecting severe fibrosis F3 and F4 was significantly higher than ARFI $(p<0.05)$. The Sp of both methods ARFI and 2D-SWE were similar, increasing with the severity of fibrosis. Both techniques can discriminate between paediatric patients with mild and severe fibrosis.

Initial comparative statistical analysis included the entire lot of 51 children with valid measurements. Kappa correlation factor was computed in order to assess the concordance between TE/ARFI and TE/2D-SWE. The results are depicted in table IV.

Further, the study group was divided in two subgroups based on SR values from TE measurements. The first subgroup included 11 children with valid measurements, but less satisfactory technical parameters: $\mathrm{SR}=60 \%-70 \%$ and/or IQR $=30 \%$. The second subgroup consisted of 40 subjects with SR between $70 \%$ and $100 \%$ 
Table V. Kappa correlation factor between TE/ARFI and TE/2D-SWE in the first and second subgroup.

\begin{tabular}{|c|c|c|}
\hline \multirow[t]{2}{*}{$\begin{array}{l}1^{\text {st }} \text { Subgroup } \\
(\mathrm{SR}=60 \%-70 \% \text { and } / \text { or } \mathrm{IQR}=30 \%)\end{array}$} & Kappa factor TE/ARFI & $\begin{array}{l}0.172, p=0.452 \\
99 \% \text { CI }[0.439-0.465]\end{array}$ \\
\hline & Kappa factor TE/2D-SWE & $\begin{array}{l}0.758, \mathrm{p}=0.001 \\
99 \% \text { CI }[0.000-0.001]\end{array}$ \\
\hline \multirow[t]{2}{*}{$\begin{array}{l}2^{\text {nd }} \text { Subgroup } \\
(\mathrm{SR}>70 \% \text { and } \mathrm{IQR}<30 \%)\end{array}$} & Kappa factor TE/ARFI & $\begin{array}{l}0.761, \mathrm{p}=0.001 \\
99 \% \text { CI }[0.000-0.001]\end{array}$ \\
\hline & Kappa factor for TE/2D-SWE & $\begin{array}{l}0.860, \mathrm{p}=0.001 \\
99 \% \text { CI }[0.000-0.001]\end{array}$ \\
\hline
\end{tabular}

TE - Transient elastography, ARFI - Acoustic Radiation Force Impulse Elastography, 2D-SWE - 2D-Shear Wave Elastography, SR - success rate, IQ - interquartile range

and IQR $<30 \%$. Advanced comparative statistical analysis was performed. Kappa Statistics was applied in order to assess the concordance between TE/ARFI and TE/2DSWE in both subgroups (table V).

\section{Discussions}

Most of the studies that have compared liver biopsies with different elastographic methods were performed on an adult population; there are only few studies performed on children [18]. A recent paediatric study compared TE with liver biopsies in a cohort of children with chronic $\mathrm{C}$ hepatitis, in order to elaborate the specific cut off values for this technique in paediatric patients [19], and another paediatric study focused on the performance of TE for evaluating liver fibrosis in infants with biliary atresia [20]. Recently, 2D-SWE accuracy was evaluated in children post liver transplantation [21]. Other studies analyzed the influence of some technical parameters on measurement reliability such as: type of probe used, place and depth of measurement $[15,22]$. Tutar et al [23] showed that although liver fibrosis can be assessed using 2D-SWE in children, differentiation of mild to moderate fibrosis stages (F1, F2) could not be achieved. The presence of steatosis significantly increased the mean 2D-SWE values on elastography and care should be taken when assessing children with nonalcoholic steatohepatitis [23].

Further paediatric studies should define different cutoff range values for discriminating between different fibrosis stages for each available elastographic method according to the underlying condition [24].

To our knowledge, this is the first direct comparison of TE, ARFI, and 2D-SWE among paediatric patients. Our study tried to validate alternative elastographic techniques with similar accuracy to TE in children and adolescents. The advantage of ARFI and 2D-SWE is highlighted by the fact that these ultrasound based elastographic methods are integrated in an ultrasound machine, that can be used for others examinations, such as gray scale ultrasound, Doppler, Contrast Enhanced Ul- trasonography (CEUS) [25]. FibroScan is an expensive machine that can be used only for LS evaluation and more recent for steatosis assessment in the liver using Controlled Attenuation Parameter (CAP) [26].

Children with narrow intercostal spaces may have high failure rates when performing TE with a standard $\mathrm{M}$ probe. The paediatric $\mathrm{S}$ probes can improve reliability in this regard. A recent study [22] aimed at addressing points of consideration in the probe choice and interpretation of measured LS by comparing FibroScan S1, S2 and $\mathrm{M}$ probes in children with biliary atresia. The authors concluded that the S1 probe is more appropriate for use in small children, especially those with a thorax perimeter of $<45 \mathrm{~cm}$, but if no $\mathrm{S}$ probe is available, the M probe may be acceptable in children whose thorax perimeter is $>45 \mathrm{~cm}[22]$.

Because BMI is both age-and sex-specific for children, we used the BMI-for-age percentile charts. All obese children with BMI above 95th percentile were assessed by FibroScan using XL probe. Compared with the $\mathrm{M}$ probe, the $\mathrm{XL}$ probe reduces TE failure and facilitates reliable LS measurements in obese patients [27]. There are no studies regarding the use of the $\mathrm{XL}$ probe in obese paediatric subjects, but according to recent studies performed on the adult population [27], we used the XL probe for obese children in this study.

Bota et al [28] published a meta-analysis on an adult population that compared the diagnostic performance of ARFI elastography and TE for the assessment of liver fibrosis using liver biopsy as the ,gold standard”. The results showed that for detection of significant fibrosis ( $\mathrm{F}$ $\geq 2$ ), the summary sensitivity was 0.74 and specificity was 0.83 for ARFI, while for TE the sensitivity was 0.78 and specificity was 0.84 . For the diagnosis of cirrhosis, the summary sensitivity was 0.87 and specificity was 0.87 for ARFI elastography and respectively 0.89 and 0.87 for TE. Inability to obtain reliable measurements was higher for TE compared to ARFI [28].

Another study published by Sporea et al [29] compared three elastographic methods TE, ARFI and 2D-SWE on 
332 adult patients with respect to the feasibility of their use for liver fibrosis evaluation. In this study, a significantly higher percentage of reliable measurements were obtained for ARFI than for TE and 2D-SWE (92.1\% versus $72.2 \%$ , $p<0.0001$ and $92.1 \%$ versus $71.3 \%, p<0.0001$ ) [29].

It seems that among adult patients, ARFI showed a higher rate of reliable measurements compared to TE or 2D-SWE. In our study, the proportion of valid measurements by means of TE using FibroScan was $94.4 \%$ and all paediatric patients with valid TE measurements had also valid results when performing ARFI and 2D-SWE elastography, with SR of $100 \%$ for all ARFI determinations. ARFI measurements correlated significantly with TE values only in those cases with SR $>70 \%$ by FibroScan. In contrast with the adult studies cited above, 2D-SWE showed better correlation with TE measurements compared to ARFI in this paediatric population. Although valid, TE measurements with SR between $60 \%$ and $70 \%$ might be less reliable in paediatric subjects, regarding the fact that they did not correlate with ARFI, only with 2D-SWE measurements. ARFI imaging and 2D-SWE had similar accuracy with TE in the majority of adult population based studies. The main technical requirement when performing ARFI is to hold breathing for several seconds and usually the adult patients cooperate better than children. Frequent body movements and the inability for young children to perform and keep apnea could decrease the accuracy of this elastographic method, explaining why ARFI values did not correlate with TE and had a lower sensitivity for detecting severe fibrosis compared to 2D-SWE. 2D-SWE can assess LS even without apnea and through very small intercostal spaces, or from the epigastrium, making the paediatric diagnostic process easier and more manageable.

The availability of liver biopsies (the main limitation of the study) in less than half of the cases enrolled in this study has made the comparison between different elastographic methods and the gold standard assessment of liver fibrosis-liver histology not feasible. Therefore, we considered TE by FibroScan the standard referrence method. Another limitation of this study was the fact that we used the $\mathrm{M}$ probe due to a practical issue: - the lack of a $\mathrm{S}$ probe. In order to avoid the use of the $\mathrm{M}$ probe as a bias factor, we enrolled in this study children with a mean age of 8.6 years $( \pm 2.5)$, weighing more than $20 \mathrm{~kg}$ and with thorax perimeter $>45 \mathrm{~cm}$.

Another limitation is related to the heterogeneous etiology of liver injuries in children and adolescents enrolled in this study. We used the cut-off range values for fibrosis staging for each elastographic technique according to the most recently published studies on an adult population, because there are no studies in children regarding the differences of staging fibrosis in different liver conditions.

\section{Conclusions}

2D-SWE based on SSI is a new technique designed to overcome some of the disadvantages of other elastographic techniques in children. Overall, 2D-SWE seems to correlate better with TE compared to ARFI in children and adolescents. The specificity of 2D-SWE and ARFI increases with the severity of fibrosis. Excluding patients with less satisfactory technical parameters for TE ( $\mathrm{SR}=60 \%-70 \%$ and/or $\mathrm{IQR}=30 \%$ ), we obtained significant correlations between all three elastographic techniques. Both 2D-SWE and ARFI are non-invasive techniques feasible to perform on children and adolescents along with TE by FibroScan.

Acknowledgment: Thanks to Dr Madalina Popescu and Dr Nadia Cornu which provided technical assistance with FibroScan, ARFI and 2D-SWE measurements.

Conflict of interest: none

\section{References}

1. European Association for the Study of the Liver. EASL clinical practice guidelines: Management of chronic hepatitis B virus infection. J Hepatol 2012; 57: 167-185.

2. Bota S, Sporea I, Şirli R, Popescu A, Dănilă M, Şendroiu $M$. Factors that influence the correlation of acoustic radiation force impulse (ARFI) elastography with liver fibrosis. Med Ultrason 2011; 13: 135-140.

3. Huang Z, Zheng J, Zeng J, Wang X, Wu T, Zheng R. Normal liver stiffness in healthy adults assessed by real-time Shear wave elastography and factors that influence this method. Ultrasound Med Biol 2014; 40: 2549-2555.

4. Wilder J, Patel K. The clinical utility of FibroScan as a noninvasive diagnostic test for liver disease. Med Devices (Auckl) 2014; 3: 107-114.

5. Trovato FM, Atzori S, Musumeci G, et al. Liver and spleen transient elastography and Acoustic Radiation Force Impulse Measurements. Performance and comparison of measurements in the same area concurrently assessed for liver fibrosis by biopsy. Adv Med Sci 2015; 60: 300-306.

6. Bavu E, Gennisson JL, Couade M, et al. Noninvasive in vivo liver fibrosis evaluation using supersonic shear imaging: a clinical study on 113 hepatitis $\mathrm{C}$ virus patients. Ultrasound Med Biol 2011; 37: 1361-1373.

7. Leschied JR, Dillman JR, Bilhartz J, Heider A, Smith EA, Lopez MJ. Shear wave elastography helps differentiate biliary atresia from other neonatal/infantile liver diseases. Pediatr Radiol 2015; 45: 366-375.

8. Gherlan GS. Liver ultrasound elastography: More than staging the disease. World J Hepatol 2015; 7: 1595-1600. 
9. Sandrin L, Fourquet B, Hasquenoph JM, et al. Transient elastography: a new noninvasive method for assessment of hepatic fibrosis. Ultrasound Med Biol 2003; 29: 17051713.

10. Friedrich-Rust M, Schwarz A, Ong M, et al. Real-time tissue elastography versus FibroScan for noninvasive assessment of liver fibrosis in chronic liver disease. Ultraschall in Med 2009; 30: 478-484.

11. Tsochatzis EA, Gurusamy KS, Ntaoula S, Cholongitas E, Davidson BR, Burroughs AK. Elastography for the diagnosis of severity of fibrosis in chronic liver disease: a metaanalysis of diagnostic accuracy. J Hepatol 2011; 54: 650659.

12. Wong GL, Chan HL, Yu Z, Chan HY, Tse CH, Wong VW. Liver fibrosis progression in chronic hepatitis B patients positive for hepatitis $\mathrm{B}$ e antigen: a prospective cohort study with paired transient elastography examination. J Gastroenterol Hepatol 2013; 28: 1762-1769.

13. Nishikawa T, Hashimoto S, Kawabe N, et al. Factors correlating with acoustic radiation force impulse elastography in chronic hepatitis C. World J Gastroenterol 2014; 20: 12891297.

14. Liao LY, Kuo KL, Chiang HS, Lin CZ, Lin YP, Lin CL. Acoustic radiation force impulse elastography of the liver in healthy patients: test location, reference range and influence of gender and body mass index. Ultrasound Med Biol 2015; 41: 698-704.

15. Fontanilla T, Cañas T, Macia A, et al. Normal values of liver shear wave velocity in healthy children assessed by acoustic radiation force impulse imaging using a convex probe and a linear probe. Ultrasound Med Biol 2014; 40: 470-477.

16. Hanquinet S, Courvoisier D, Kanavaki A, Dhouib A, Anooshiravani M. Acoustic radiation force impulse imagingnormal values of liver stiffness in healthy children. Pediatr Radiol 2013; 43: 539-544.

17. Sporea I, Gilja OH, Bota S, Şirli R, Popescu A. Liver Elastography - An Update. Med Ultrason 2013; 15: 304-314.

18. Shen QL, Chen YJ, Wang ZM, et al. Assessment of liver fibrosis by Fibroscan as compared to liver biopsy in biliary atresia. World J Gastroenterol 2015; 21: 6931-6936.
19. Awad Mel-D, Shiha GE, Sallam FA, Mohamed A, El-Tawab A. Evaluation of liver stiffness measurement by FibroScan as compared to liver biopsy for assessment of hepatic fibrosis in children with chronic hepatitis C. J Egypt Soc Parasitol 2013; 43: 805-819.

20. Shin NY, Kim MJ, Lee MJ, et al. Transient elastography and sonography for prediction of liver fibrosis in infants with biliary atresia. J Ultrasound Med 2014; 33: 853-864.

21. Yoon JH, Lee JY, Woo HS, et al. Shear wave elastography in the evaluation of rejection or recurrent hepatitis after liver transplantation. Eur Radiol 2013; 23: 1729-1737.

22. Kim S, Kang Y, Lee MJ, Kim MJ, Han SJ, Koh H. Points to be considered when applying FibroScan $\mathrm{S}$ probe in children with biliary atresia. J Pediatr Gastroenterol Nutr 2014; 59: 624-628.

23. Tutar O, Beşer ÖF, Adaletli I, et al. Shear wave elastography in the evaluation of liver fibrosis in children. J Pediatr Gastroenterol Nutr 2014; 58: 750-755.

24. Göbel T, Schadewaldt-Tümmers J, Greiner L, Poremba C, Häussinger D, Erhardt A. Transient elastography improves detection of liver cirrhosis compared to routine screening tests. World J Gastroenterol 2015; 21: 953-960.

25. Zheng J, Guo H, Zeng J, et al. Two-dimensional shear-wave elastography and conventional US: the optimal evaluation of liver fibrosis and cirrhosis. Radiology 2015; 275: 290300.

26. Mi YQ, Shi QY, Xu L, et al. Controlled attenuation parameter for noninvasive assessment of hepatic steatosis using Fibroscan: validation in chronic hepatitis B. Dig Dis Sci 2015; 60: 243-251.

27. Myers RP, Pomier-Layrargues G, Kirsch R, et al. Feasibility and diagnostic performance of the FibroScan XL probe for liver stiffness measurement in overweight and obese patients. Hepatology 2012; 55:199-208.

28. Bota S, Herkner H, Sporea I, et al. Meta-analysis: ARFI elastography versus transient elastography for the evaluation of liver fibrosis, Liver Int 2013; 33: 1138-1147.

29. Sporea I, Bota S, Jurchis A, et al. Acoustic radiation force impulse and supersonic shear imaging versus transient elastography for liver fibrosis assessment. Ultrasound Med Biol 2013; 39: 1933-1941. 